\title{
Efeitos do consumo de leite de vaca pela criança antes do primeiro ano de vida
}

\author{
Effects of consumption of cow's millk by the child before the first year of life \\ Efectos del consumo de leche de vaca por el niño antes del primer año de vida
}

Yanca Curty Ribeiro Christoff Ornelas

ORCID: https://orcid.org/0000-0001-5003-387X

Faculdades Unidas do Norte de Minas Gerais, Brasil

E-mail: yancacurty@ hotmail.com

Silvania Paiva dos Santos

ORCID: https://orcid.org/0000-0002-6911-8256

Universidade Estadual de Montes Claros, Brasil

E-mail: silvaniapaivasantos@gmail.com.br

Ely Carlos Pereira de Jesus

ORCID: https://orcid.org/0000-0003-2071-6287

Faculdades Unidas do Norte de Minas Gerais, Brasil

E-mail: elycarlopereira@yahoo.com.br

Anderson Neco Rocha

ORCID: https://orcid.org/0000-0002-5372-7786

Faculdades Santo Agostinho, Brasil

E-mail: nrandrson@gmail.com

Richard Rennan Soares Barbosa

ORCID: https://orcid.org/0000-0002-9801-6371

Faculdades Unidas do Norte de Minas Gerais, Brasil

E-mail: richardrennansoaresbarbosa@gmail.com

Suelen Ferreira Rocha

ORCID: https://orcid.org/0000-0003-3197-3350

Universidade Estadual de Montes Claros, Brasil

E-mail: suellenfrocha@ hotmail.com

Karla Talita Santos Silva

ORCID: https://orcid.org/0000-0001-5636-1655

Universidade Federal de Minas Gerais, Brasil

E-mail: ktalitass@hotmail.com

Sylmara Corrêa Monteiro

ORCID: https://orcid.org/0000-0003-4546-336X Instituto Federal de Educação, Ciência e Tecnologia do Norte de Minas Gerais, Brasil E-mail: scmenfermagem9@gmail.com

Bruno de Pinho Amaral

ORCID: https://orcid.org/0000-0002-1515-8877

Universidade Estadual de Montes Claros, Brasil

E-mail: brunophelp@hotmail.com

Joana Carolina Rodrigues dos Santos Schramm

ORCID: https://orcid.org/0000-0001-6257-9546

Universidade Estadual de Montes Claros, Brasil

E-mail: jocarol2@yahoo.com.br

Tatiane Pereira Horta

ORCID: https://orcid.org/0000-0002-0521-0416

Faculdade de Saúde e Humanidades Ibituruna, Brasil

E-mail: tatihorta111@gmail.com

Andreia Correia

ORCID: https://orcid.org/0000-0002-8936-2459

Faculdades Unidas do Norte de Minas Gerais, Brasil

E-mail: deia3456@gmail.com

Samara Monteiro Rodrigues

ORCID: https://orcid.org/0000-0001-6679-5983

Faculdades Unidas do Norte de Minas Gerais, Brasil

E-mail: samaraenf4@gmail.com

Isabella Batista Vieira

ORCID: https://orcid.org/0000-0002-0284-2338

Faculdades Unidas do Norte de Minas Gerais, Brasil

E-mail: isabellabatistavieira@hotmail.com

Andressa Ferreira Costa

ORCID: https://orcid.org/0000-0002-0304-7600

Faculdades Unidas do Norte de Minas Gerais, Brasil

E-mail: andressacosta@hotmail.com 


\title{
Resumo
}

Introdução: A alimentação infantil é um fator essencial para o crescimento e desenvolvimento plenos da criança. Objetivo: analisar os efeitos da introdução do leite de vaca na alimentação da criança antes de um ano de vida. Materiais e Métodos: Trata-se de uma revisão integrativa da literatura, desenvolvida a partir de pesquisa na base de dados LILACS, MEDLINE, PubMed, Biblioteca virtual em saúde e Scielo, a partir dos descritores: aleitamento materno, desmame precoce, leite de vaca e amamentação exclusiva. Considerou-se como critérios de inclusão estudos que abordassem os fatores relacionados à alimentação infantil e os efeitos do desmame precoce e da inserção do leite de vaca na saúde e no desenvolvimento adequado das crianças menores de um ano de idade, artigos escritos em português, publicados a partir do ano de 2008 até 2021, disponíveis eletronicamente. Considerações finais: O leite de vaca, não é o mais recomendável para a alimentação da criança antes de um ano de vida, pois além de não suprir as necessidades nutricionais da criança pode desencadear alergias e intolerâncias alimentares, doenças crônicas como obesidade e diabetes mellitus e anemia ferropriva.

Palavras-chave: Aleitamento materno; Desmame precoce; Leite de vaca; Amamentação exclusiva; Substitutos do leite humano.

\begin{abstract}
Introduction: Infant feeding is an essential factor for the full growth and development of the child. Objective: to analyze the effects of the introduction of cow's milk on the child's diet before one year of life. Materials and Methods: This is an integrative review of the literature, developed from research in lilacs, MEDLINE, PubMed, virtual health library and Scielo, from the descriptors: breastfeeding, early weaning, cow's milk and exclusive breastfeeding. Inclusion criteria were studies addressing factors related to infant feeding and the effects of early weaning and the insertion of cow's milk in the health and adequate development of children under one year of age, articles written in Portuguese, published from 2008 to 2021, available electronically. Final considerations: Cow's milk is not the most recommended for feeding the child before a year of life, because in addition to not meeting the nutritional needs of the child can trigger allergies and food intolerances, chronic diseases such as obesity and diabetes mellitus and iron deficiency anemia.
\end{abstract}

Keywords: Maternal lactation; Early weaning; Cow's milk; Exclusive maternal lactation; Human milk substitutes.

\begin{abstract}
Resumen
Introducción: La alimentación infantil es un factor esencial para el pleno crecimiento y desarrollo del niño. Objetivo: analizar los efectos de la introducción de la leche de vaca en la dieta del niño antes de un año de vida. Materiales y Métodos: Se trata de una revisión integradora de la literatura, desarrollada a partir de investigaciones en lilas, MEDLINE, PubMed, biblioteca virtual de salud y Scielo, a partir de los descriptores: lactancia materna, destete precoz, leche de vaca y lactancia materna exclusiva. Los criterios de inclusión fueron estudios que abordaron los factores relacionados con la alimentación infantil y los efectos del destete temprano y la inserción de la leche de vaca en la salud y el desarrollo adecuado de los niños menores de un año, artículos escritos en portugués, publicados de 2008 a 2021, disponibles electrónicamente. Consideraciones finales: La leche de vaca no es la más recomendada para alimentar al niño antes de un año de vida, ya que además de no satisfacer las necesidades nutricionales del niño puede desencadenar alergias e intolerancias alimentarias, enfermedades crónicas como la obesidad y la diabetes mellitus y la anemia ferropénica.
\end{abstract}

Palabras clave: Lactancia materna; Destete temprano; Leche de vaca; Lactancia materna exclusiva; Sustitutos de la leche humana.

\section{Introdução}

A alimentação é um dos fatores que mais interfere na saúde da criança. A primeira infância é um estágio da vida biologicamente vulnerável, logo, uma alimentação e nutrição adequadas são fundamentais para garantir a manutenção da saúde, do crescimento e desenvolvimento infantil.

Especificamente para a criança, os primeiros anos de vida de são caracterizados pela velocidade de crescimento e desenvolvimento, para garantir que aconteçam adequadamente a alimentação exerce um papel primordial (OMS, 2013). Sendo a primeira infância uma das fases mais vulneráveis, a qualidade e a quantidade dos alimentos consumidos são aspectos importantes a serem observados, pois repercutiram ao longo da vida, sendo necessário levar em consideração também as deficiências e distúrbios nutricionais (Walker et al., 2007; Cunha, Leite et al., 2015; Victora et al, 2016). 
A Sociedade Brasileira de Pediatria (2012) destaca a importância da nutrição infantil quando ressalta que a alimentação, por ser simples e cotidiana, é reconhecida como uma das principais maneiras de manter a saúde, sendo de fundamental relevância para o desenvolvimento do bebê tanto na vida gestacional quanto extra-uterina (Sociedade Brasileira de Pediatria, 2012).

O Ministério da Saúde recomenda que crianças amamentem exclusivamente no peito até os seis meses de vida e, após esse período, deverá ser dada alimentação complementar apropriada, sem parar com a amamentação até pelo menos 2 anos de idade. A continuidade do aleitamento materno até os dois anos ou mais é muito importante, porque garante a suplementação de micronutrientes da alimentação, como por exemplo, do ferro (Brasil, 2014).

Entretanto, apesar da simplicidade do ato de amamentar, pesquisas têm registrado, no Brasil, a baixa prevalência do aleitamento materno exclusivo até os seis meses, com o leite materno sendo substituído pelo leite de vaca que, além de não suprir as necessidades de ferro e de outros nutrientes essenciais, ainda mostra-se como considerável fator de risco para o desenvolvimento adequado das crianças. Quando adotado na alimentação infantil antes de um ano de vida, o leite de vaca pode ser um determinante de algumas doenças como diabetes mellitus tipo 1 e alergias alimentares (Pereira, Alfenas, Araújo, 2014). Levando em consideração a alimentação adequada da criança, esta introdução antecipada pode levar a consequências que são prejudiciais à saúde, principalmente quando a mesma é realizada antes de completar o desenvolvimento fisiológico (Organização Mundial da Saúde, 2013).

No aspecto nutricional, torna-se inviável pois aumenta os riscos inerentes à criança, podendo causar contaminações, alergias, diminuição na absorção de outros nutrientes, uma vez que, de acordo estudos experimentais, o leite de vaca tem a capacidade de inibir a absorção de ferro nos demais alimentos ingeridos pela criança, podendo elevar o risco de anemia e implicar no risco do desmame precoce (Agostini et al, 2008).

Sabendo-se da importância do consumo alimentar adequado na primeira infância, em especial com a adoção do aleitamento materno exclusivo até os seis meses de idade para o alcance das necessidades nutricionais da criança e levando em consideração que o leite de vaca tem sido cada vez mais cedo utilizado como substituto do leite materno, este artigo de revisão tem como objetivo analisar através dos trabalhos existentes os efeitos da introdução do leite de vaca na alimentação da criança antes de um ano de vida

\section{Metodologia}

Trata-se de um estudo tipo revisão integrativa da literatura que, inspirada em Mendes, Silveira, \& Galvão (2012), percorreu as seguintes etapas: estabelecimento da hipótese e objetivos da revisão integrativa, estabelecimento de critérios de inclusão e exclusão de artigos (seleção da amostra), definição das informações a serem extraídas dos artigos selecionados, análise dos resultados, discussão e apresentação dos resultados, e a última etapa foi constituída pela apresentação da revisão.

Para tanto, considerou-se como critérios de inclusão estudos que abordassem os fatores relacionados à alimentação infantil e os efeitos do desmame precoce e da inserção do leite de vaca na saúde e desenvolvimento adequado das crianças menores de um ano de idade, artigos escritos em português, publicados a partir do ano de 2008 até 2021, disponíveis eletronicamente. Como critérios de exclusão foram considerados os artigos científicos publicados até 2007.

Realizou-se busca bibliográfica na base de dados LILACS (Literatura Latino-Americana e do Caribe em Ciências da Saúde) do Centro Latino-Americano; MEDLINE (Medical Literature Analysis and Retrieval System Online), PubMed, Biblioteca virtual em saúde (BVS) e Scielo, a partir dos descritores: aleitamento materno, desmame precoce, leite de vaca e amamentação exclusiva, substitutos do leite humano.

Para a coleta de dados foi elaborado e utilizado, uma matriz de síntese, instrumento adaptado para as peculiaridades da temática pesquisada. $\mathrm{O}$ instrumento apresenta as seguintes informações: identificação do artigo e autores, fonte de localização, objetivos, delineamento, características do estudo, população/amostra, resultados e considerações finais. Na Figura a seguir, 
apresenta-se o processo de seleção dos artigos (Figura 1).

Figura 1. Processo de seleção dos artigos.

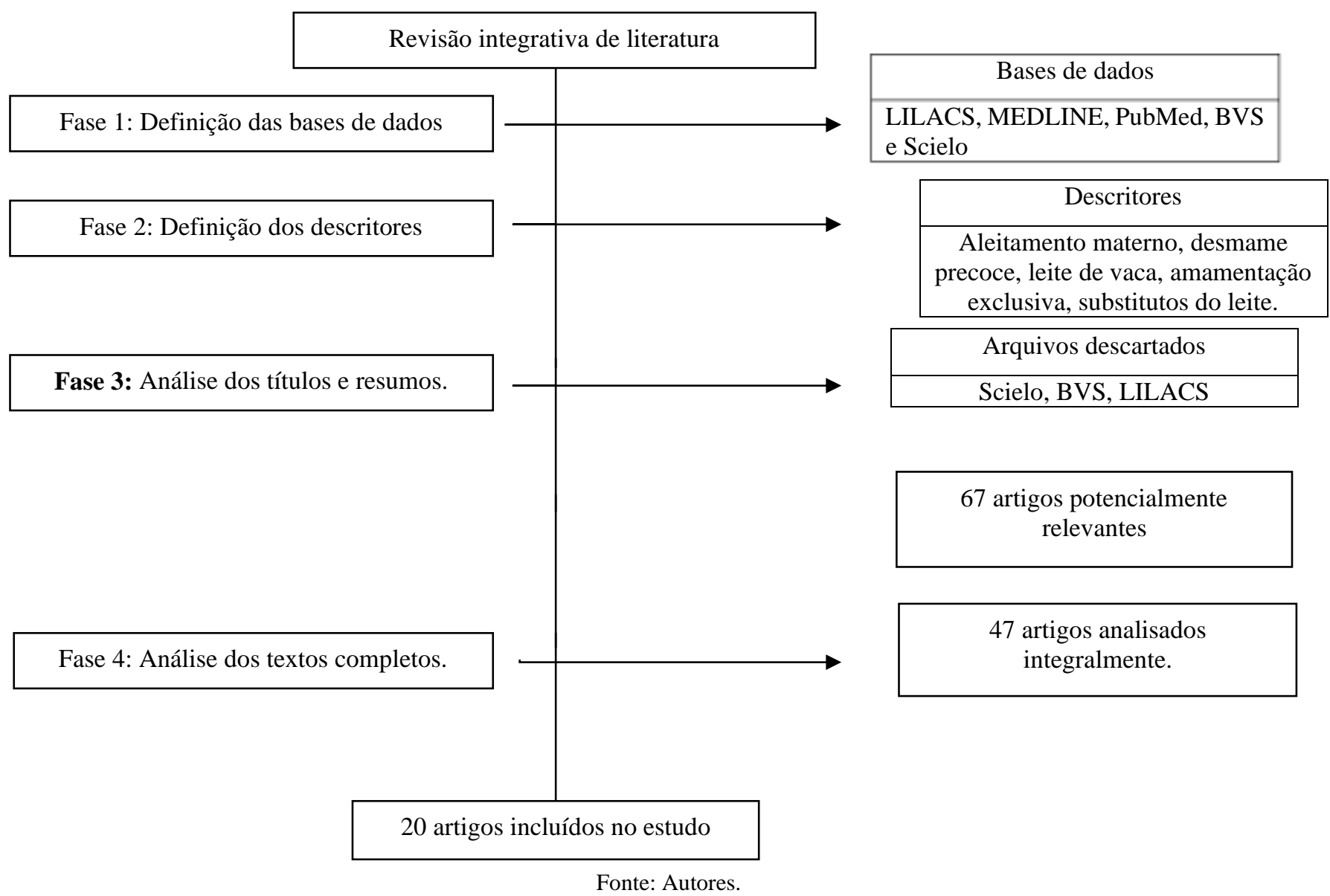

\section{Resultados e Discussão}

Foram encontrados 20 artigos que versavam sobre o tema da pesquisa, dos quais oito foram excluídos por terem sido publicados no ano anterior a 2008, foram excluídos ainda os estudos duplicados nas bases de dados secundários. Dentro dos critérios de inclusão estabelecidos previamente, foram selecionados 20 artigos, encontrados na base de dados SCIELO, LILACS, MEDLINE e em Revistas de Pediatria, Nutrição e Enfermagem todos dispostos no Quadro 1. 
Research, Society and Development, v. 11, n. 3, e41311325554, 2022

(CC BY 4.0) | ISSN 2525-3409 | DOI: http://dx.doi.org/10.33448/rsd-v11i3.25554

Quadro 1. Distribuição do conteúdo dos artigos analisados segundo autores, ano de publicação, objetivos, delineamento, população estudada e principais resultados.

\begin{tabular}{|c|c|c|c|c|}
\hline & $\begin{array}{l}\text { Autores/ Ano de } \\
\text { Publicação }\end{array}$ & Objetivos & Delineamento & Principais resultados \\
\hline 1 & $\begin{array}{l}\text { PEREIRA, } \\
\text { ALFENAS, } \\
\text { ARAÚJO } \\
(2014)\end{array}$ & $\begin{array}{l}\text { Realizar uma análise crítica da literatura } \\
\text { para avaliar a influência da amamentação } \\
\text { no risco do desenvolvimento de diabetes } \\
\text { mellitus. }\end{array}$ & $\begin{array}{l}\text { Revisão não sistemática nas bases de dados } \\
\text { Scielo, Lilacs, MedLine. }\end{array}$ & A ausência de amamentação é um possível fator de risco modificável para diabetes tipo 1 e 2 . \\
\hline 2 & $\begin{array}{l}\text { DIAS, FREIRE, } \\
\text { FRANCESCHIN } \\
\text { I (2010) }\end{array}$ & $\begin{array}{l}\text { Revisar as recomendações atuais sobre a } \\
\text { alimentação complementar de crianças } \\
\text { menores de dois anos }\end{array}$ & $\begin{array}{l}\text { Revisão de literatura nas bases de dados } \\
\text { SciELO, MedLine e Lilacs, livros técnicos e } \\
\text { publicações de órgãos nacionais e } \\
\text { internacionais. }\end{array}$ & $\begin{array}{l}\text { A introdução da alimentação complementar é uma etapa crítica e vários são os prejuízos do } \\
\text { aleitamento artificial e da introdução precoce e/ou inadequada dos alimentos. }\end{array}$ \\
\hline 3 & $\begin{array}{l}\text { SCHINCAGLIA } \\
\text { et al (2015) }\end{array}$ & $\begin{array}{l}\text { Analisar as práticas alimentares e fatores } \\
\text { associados à introdução precoce da } \\
\text { alimentação complementar entre crianças } \\
\text { menores de seis meses, nascidas em } \\
\text { maternidade na região noroeste de } \\
\text { Goiânia, Goiás, Brasil. }\end{array}$ & $\begin{array}{l}\text { Estudo transversal, realizado de agosto de } \\
2005 \text { a fevereiro de } 2007 \text {. A amostra foi } \\
\text { composta por } 362 \text { recém-nascidos de ambos } \\
\text { os sexos. }\end{array}$ & $\begin{array}{l}\text { A prevalência da introdução precoce da alimentação complementar foi elevada, associada a fatores } \\
\text { ambientais e gestacionais. }\end{array}$ \\
\hline 4 & $\begin{array}{l}\text { CAMPAGNOLO } \\
\text { et al (2012) }\end{array}$ & $\begin{array}{l}\text { Investigar a adequação das práticas } \\
\text { alimentares no primeiro ano de vida e seus } \\
\text { fatores associados na cidade de Porto } \\
\text { Alegre (RS). }\end{array}$ & $\begin{array}{l}\text { Estudo transversal foi realizado durante a } \\
\text { Campanha Nacional de Imunização no ano de } \\
\text { 2008. Utilizou-se amostragem por } \\
\text { conglomerados em dois estágios e foram } \\
\text { avaliadas } 1.099 \text { crianças menores de um ano } \\
\text { de idade, em } 31 \text { postos de vacinação. }\end{array}$ & $\begin{array}{l}\text { Este estudo mostrou a existência de fatores de risco para a interrupção precoce do aleitamento } \\
\text { materno exclusivo e o consumo de alimentos inadequados aos lactentes. }\end{array}$ \\
\hline 5 & JOSÉ et al (2016) & $\begin{array}{l}\text { Investigar na literatura científica as } \\
\text { relações entre a hipersensibilidade } \\
\text { alimentar com o desmame precoce, } \\
\text { verificando a associação entre esses dois } \\
\text { conceitos, abordando as principais causas } \\
\text { que levam ao desenvolvimento de alergias } \\
\text { alimentares. }\end{array}$ & $\begin{array}{l}\text { Revisão de literatura nas bases de dados } \\
\text { Scielo, Lilacs, Science Direct, PubMed e } \\
\text { MedLine. Artigos dos últimos } 15 \text { anos, } \\
\text { referente à relação do desmame precoce e } \\
\text { reações alergênicas. }\end{array}$ & $\begin{array}{l}\text { Verificou-se que os lactentes, principalmente, nos primeiros meses de vida apresentam o sistema } \\
\text { imunológico e gastrointestinal imaturo, estando mais susceptíveis a absorção de macromoléculas } \\
\text { e ao desenvolvimento de reações de hipersensibilidade, que podem desencadear alergias } \\
\text { alimentares. }\end{array}$ \\
\hline 6 & $\begin{array}{l}\text { GIULIANI et al } \\
\text { (2011) }\end{array}$ & $\begin{array}{l}\text { Verificar a prevalência de aleitamento } \\
\text { materno (AM), aleitamento materno } \\
\text { exclusivo (AME) e AM predominante } \\
\text { (AMP) até } 6 \text { meses e, do início do } \\
\text { desmame precoce (AME) }\end{array}$ & $\begin{array}{l}\text { Estudo transversal de características } \\
\text { descritivas e analíticas, realizado a partir de } \\
\text { entrevistas guiadas por um questionário, } \\
\text { aplicado às mães entre janeiro e abril de } 2005 \text {, } \\
\text { que realizaram consultas de puericultura no } \\
\text { Hospital Universitário da Universidade } \\
\text { Federal de Santa Catarina (HU - UFSC) e na } \\
\text { Unidade de Saúde do Saco Grande II }\end{array}$ & $\begin{array}{l}\text { O aleitamento materno foi realizado por } 98 \% \text { da amostra; entre as que amamentaram } 18,4 \% \\
\text { realizaram aleitamento materno exclusivo até o sexto mês de vida do seu filho. O início do } \\
\text { desmame precoce foi realizado por } 81,6 \% \text { das participantes sendo que, destas, } 15 \% \text { realizaram } \\
\text { aleitamento materno predominante nos } 6 \text { meses após a gestação. }\end{array}$ \\
\hline
\end{tabular}


Research, Society and Development, v. 11, n. 3, e41311325554, 2022

(CC BY 4.0) | ISSN 2525-3409 | DOI: http://dx.doi.org/10.33448/rsd-v11i3.25554

\begin{tabular}{|c|c|c|c|c|}
\hline & & & $\begin{array}{l}\text { (USSGII), totalizando uma amostra de } 200 \\
\text { mães. }\end{array}$ & \\
\hline 7 & $\begin{array}{lll}\text { LEAL } & \text { et } & a l \\
(2011) & & \end{array}$ & $\begin{array}{l}\text { Estimar a prevalência de anemia e } \\
\text { identificar seus fatores associados em } \\
\text { crianças de seis a } 59 \text { meses. }\end{array}$ & $\begin{array}{l}\text { Estudo transversal com dados da III Pesquisa } \\
\text { Estadual de Saúde e Nutrição/Pernambuco. } \\
\text { Amostra representativa de } 1.403 \text { crianças } \\
\text { para as áreas urbana e rural. }\end{array}$ & $\begin{array}{l}\text { A prevalência de anemia nas crianças pernambucanas é semelhante nas áreas urbana e rural. Os } \\
\text { fatores associados à anemia apresentados devem ser considerados no planejamento de medidas } \\
\text { efetivas para o seu controle. }\end{array}$ \\
\hline 8 & OMS (2013) & $\begin{array}{l}\text { Divulgar as tendências e níveis de } \\
\text { mortalidade infantil. }\end{array}$ & $\begin{array}{l}\text { Relatório que utilizou dados da UNICEF } \\
\text { referentes à mortalidade infantil entre } 1990 \text { a } \\
2012 \text {. }\end{array}$ & $\begin{array}{l}\text { O relatório indica que, se permanecerem as atuais tendências, o mundo não atingirá o Objetivo de } \\
\text { Desenvolvimento do Milênio } 4 \text { (diminuir em dois terços a taxa de mortalidade de crianças menores } \\
\text { de } 5 \text { anos até 2015). Pior ainda: se continuarem as atuais tendências, o objetivo não será alcançado } \\
\text { até } 2028 \text {. }\end{array}$ \\
\hline 9 & $\begin{array}{l}\text { DIAS, FREIRE, } \\
\text { FRANCESCHIN } \\
\text { I (2010) }\end{array}$ & $\begin{array}{l}\text { Revisar as recomendações atuais sobre a } \\
\text { alimentação complementar de crianças } \\
\text { menores de dois anos }\end{array}$ & $\begin{array}{l}\text { Revisão de literatura realizada a partir da } \\
\text { pesquisa em banco de dados - } \\
\text { Scielo, MedLine e Lilacs e de publicações da } \\
\text { Organização Mundial da Saúde e do } \\
\text { Ministério da Saúde. Artigos publicados de } \\
2002 \text { a } 2006 \text { e idioma de redação em } \\
\text { português, inglês e espanhol. }\end{array}$ & $\begin{array}{l}\text { A introdução da alimentação complementar é uma etapa crítica e vários são os prejuízos do } \\
\text { aleitamento artificial e da introdução precoce e/ou inadequada dos alimentos. }\end{array}$ \\
\hline 10 & $\begin{array}{l}\text { OLIVEIRA, } \\
\text { FANARO (2015) }\end{array}$ & $\begin{array}{l}\text { Comparar o estado nutricional e práticas } \\
\text { alimentares de crianças de } 1 \text { a } 7 \text { anos, que } \\
\text { tiveram diferentes tipos de amamentação } \\
\text { nos seis primeiros meses de vida, a fim de } \\
\text { explanar os benefícios do aleitamento } \\
\text { materno contra alergias, sobrepeso e } \\
\text { obesidade infantil. }\end{array}$ & $\begin{array}{l}\text { Estudo transversal composto por } 40 \text { crianças } \\
\text { matriculadas nas redes particular e pública de } \\
\text { ensino infantil na cidade de São Paulo - SP. } \\
\text { Os dados foram obtidos através de } \\
\text { questionário fechado enviado aos pais ou } \\
\text { responsáveis, a fim de coletar informações } \\
\text { como tipo de aleitamento recebido (materno } \\
\text { ou fórmulas), tempo de amamentação e } \\
\text { alergias existentes na criança, bem como } \\
\text { variáveis socioeconômicas }\end{array}$ & A amamentação representa um fator de proteção contra o sobrepeso, obesidade e alergias infantis. \\
\hline 11 & $\begin{array}{l}\text { SILVA, } \\
\text { GUBERT (2010) }\end{array}$ & $\begin{array}{l}\text { Analisar as informações de sites da } \\
\text { internet sobre o aleitamento materno e } \\
\text { alimentação complementar de acordo com } \\
\text { a adequação às recomendações do } \\
\text { Ministério da Saúde do Brasil. }\end{array}$ & $\begin{array}{l}\text { Estudo transversal, com amostra de } \\
103 \text { sites de profissionais de saúde. } \\
\text { Analisaram-se informações sobre aleitamento } \\
\text { materno (AM) e alimentação complementar } \\
\text { (AC). As informações foram quantificadas e } \\
\text { analisadas qualitativamente. }\end{array}$ & $\begin{array}{l}\text { A maior parte das informações sobre aleitamento materno está de acordo com o preconizado pelo } \\
\text { Ministério da Saúde, mas são insuficientes para estimular a prática do aleitamento materno. Já as } \\
\text { informações sobre alimentação complementar na sua maioria divergem do recomendado. }\end{array}$ \\
\hline 12 & $\begin{array}{l}\text { MENDONÇA, } \\
\text { COCCO, SAMI, } \\
\text { SOLÉ (2011) }\end{array}$ & $\begin{array}{l}\text { Revisar os principais protocolos de } \\
\text { padronização para o teste de provocação } \\
\text { oral aberto aplicado a crianças com } \\
\text { suspeita de alergia ao leite de vaca } \\
\text { mediada por imunoglobulina E. }\end{array}$ & $\begin{array}{l}\text { Foram selecionados artigos publicados, nos } \\
\text { últimos dez anos, nas bases de dados Medline, } \\
\text { Lilacs e SciELO, utilizando-se os descritores } \\
\text { de assunto: "hipersensibilidade alimentar", } \\
\text { "leite de vaca", "alergia ao leite de vaca", } \\
\text { "teste de provocação oral", "crianças" e } \\
\text { "diagnóstico" }\end{array}$ & $\begin{array}{l}\text { Mesmo considerando as limitações que dificultam a aplicação do teste de provocação oral na } \\
\text { prática clínica, a implementação do teste nos serviços de saúde poderia reduzir diagnósticos falsos- } \\
\text { positivos de alergias. }\end{array}$ \\
\hline
\end{tabular}


Research, Society and Development, v. 11, n. 3, e41311325554, 2022

(CC BY 4.0) | ISSN 2525-3409 | DOI: http://dx.doi.org/10.33448/rsd-v11i3.25554

\begin{tabular}{|c|c|c|}
\hline 13 & $\begin{array}{l}\text { SALDANA et al } \\
\text { (2016) }\end{array}$ & $\begin{array}{l}\text { Verificar o tipo de leite consumido por } \\
\text { crianças menores de um ano de idade e } \\
\text { identificar variáveis associadas ao } \\
\text { consumo de leite não materno (LNM) - } \\
\text { fórmula infantil ou leite de vaca (LV). }\end{array}$ \\
\hline 14 & BLUMER (2015) & $\begin{array}{l}\text { Descrever as consequências do consumo } \\
\text { de leite e laticínios. }\end{array}$ \\
\hline 15 & $\begin{array}{l}\text { PASSANHA, } \\
\text { MANCUSO, } \\
\text { SILVA (2010) }\end{array}$ & $\begin{array}{l}\text { Identificar os elementos protetores do leite } \\
\text { materno que atuam na prevenção de } \\
\text { doenças gastrintestinais e respiratórias }\end{array}$ \\
\hline 16 & $\begin{array}{l}\text { SALDAN et al } \\
\text { (2017) }\end{array}$ & $\begin{array}{l}\text { Verificar o tipo de leite consumido por } \\
\text { crianças menores de um ano de idade e } \\
\text { identificar variáveis associadas ao } \\
\text { consumo de leite não materno (LNM) - } \\
\text { fórmula infantil ou leite de vaca (LV). }\end{array}$ \\
\hline
\end{tabular}

Estudo transversal realizado durante a Campanha Nacional de Vacinação contra Poliomielite 2012. Os acompanhantes de 935 crianças menores de um ano responderam a um questionário estruturado sobre a imentação da criança nas últimas 24 horas. As estimativas são apresentadas por pontos e intervalo de confiança de 95\% (IC95\%). Adotou a estatística $F$ para verificar diferenças na proporção de consumo dos tipos de leite segundo a faixa etária das crianças.

Revisão integrativa

Revisão sistemática de literatura sobre o tema, onde as informações foram obtidas por meio de rastreamento literário empregando-se a técnica booleana utilizando os seguintes descritores: leite materno and doenças gastrintestinais; e leite materno and doenças respiratórias. Utilizou-se fontes de pesquisa como publicações específicas e os provedores de pesquisa Bireme, Lilacs, Medline e Scielo. Como critérios de busca foram utilizados limites de idiomas (português, espanhol e inglês), e de período (1996 a 2009).

Estudo transversal realizado durante a Campanha Nacional de Vacinação contra Poliomielite 2012. Os acompanhantes de 935 crianças menores de um ano responderam a um questionário estruturado sobre a limentação da criança nas últimas 24 horas. As entação da criança nas últinas 24 horas. As estinativas são apresentadas por pontos e intervalo de confiança de 95\% (IC95\%). Adotou-se a estatística $\mathrm{F}$ para verificar
As crianças receberam leite de vaca precocemente (antes do primeiro ano de vida), em especial aquelas pertencentes às famílias de menor nível socioeconômico e inseridas em programa social específico para recebimento de leite.

Hoje não faltam estudos e evidências de que o consumo de leite e laticínios estão diretamente ligados à causa de diversas doenças, principalmente em crianças que fazem o uso do leite de vaca antes dos seis meses de vida. O fato é que são diversas as fontes de cálcio, vitaminas e sais minerais noutros alimentos, que são melhor absorvidos pelo organismo e não prejudicam nosso corpo como o leite de vaca.

O leite materno também possui outras imunoglobulinas, anticorpos, oligossacarídeos, lipídeos, peptídeos bioativos, entre outros constituintes exclusivos com mecanismos específicos que, além da proteção contra essas doenças, estimulam o desenvolvimento do sistema imune do lactente. Assim, nenhum outro leite possui essas propriedades e podem até ser a causa destas doenças.

As crianças receberam leite de vaca precocemente (antes do primeiro ano de vida), em especial aquelas pertencentes às famílias de menor nível socioeconômico e inseridas em programa social específico para recebimento de leite. 
Research, Society and Development, v. 11, n. 3, e41311325554, 2022

(CC BY 4.0) | ISSN 2525-3409 | DOI: http://dx.doi.org/10.33448/rsd-v11i3.25554

\begin{tabular}{|c|c|c|c|c|}
\hline & & & $\begin{array}{l}\text { diferenças na proporção de consumo dos tipos } \\
\text { de leite segundo a faixa etária das crianças } \\
\text { ( }<6 \text { meses e 6-11 meses) e a associação entre } \\
\text { o consumo de leite não materno e as variáveis } \\
\text { estudadas. }\end{array}$ & \\
\hline 17 & $\begin{array}{l}\text { AMARAL } \\
(2015)\end{array}$ & $\begin{array}{l}\text { Identificar os fatores que podem } \\
\text { influenciar as nutrizes na interrupção do } \\
\text { aleitamento materno exclusivo durante os } \\
\text { primeiros seis meses de vida do lactente }\end{array}$ & $\begin{array}{l}\text { Pesquisa descritiva, exploratória, qualitativa. } \\
\text { As informações foram coletadas por meio de } \\
\text { entrevista semiestruturada com } 14 \text { mulheres } \\
\text { que estavam em aleitamento materno e } \\
\text { realizaram pré-natal em Unidades Básicas de } \\
\text { Saúde da Família no município de Campina } \\
\text { Grande-PB, no período de abril a maio de } \\
\text { 2013. Os dados foram tratados pela técnica de } \\
\text { Análise de Conteúdo de Bardin. }\end{array}$ & $\begin{array}{l}\text { Pouco conhecimento das nutrizes em relação ao vínculo afetivo do binômio, à redução dos gastos } \\
\text { da família com a alimentação da criança e ao risco de hemorragias no pós-parto; crença na produção } \\
\text { insuficiente de leite; dificuldade de pega da mama; e diversas intercorrências mamárias no pós- } \\
\text { parto. }\end{array}$ \\
\hline 18 & $\begin{array}{l}\text { SILVA, } \\
\text { GUBERT (2010) }\end{array}$ & $\begin{array}{l}\text { Analisar as informações de sites da } \\
\text { internet sobre o aleitamento materno e } \\
\text { alimentação complementar de acordo com } \\
\text { a adequação às recomendações do } \\
\text { Ministério da Saúde do Brasil. }\end{array}$ & $\begin{array}{l}\text { Estudo transversal, com amostra de } \\
103 \text { sites de profissionais de saúde. } \\
\text { Analisaram-se informações sobre aleitamento } \\
\text { materno (AM) e alimentação complementar } \\
\text { (AC). As informações foram quantificadas e } \\
\text { analisadas qualitativamente. }\end{array}$ & $\begin{array}{l}\text { A maior parte das informações sobre aleitamento materno está de acordo com o preconizado pelo } \\
\text { Ministério da Saúde, mas são insuficientes para estimular a prática do aleitamento materno. Já as } \\
\text { informações sobre alimentação complementar na sua maioria divergem do recomendado com a } \\
\text { maior parte das mães inserindo o leite de vaca e seus derivados na alimentação da criança antes de } \\
\text { um ano de vida. }\end{array}$ \\
\hline 19 & $\begin{array}{l}\text { SILVA } \quad \text { et } \quad a l \\
(2016)\end{array}$ & $\begin{array}{l}\text { Dissertar sobre os Benefícios do } \\
\text { Aleitamento Materno - AM para o } \\
\text { Crescimento e Desenvolvimento Infantil }\end{array}$ & $\begin{array}{l}\text { Trata-se de uma revisão sistemática realizada } \\
\text { a partir de pesquisa em artigos nas bases de } \\
\text { dados:SCIELO e LILACS }\end{array}$ & $\begin{array}{l}\text { o AM é, indiscutivelmente, o melhor alimento a ser ofertado, pois oferece a quantidade energética } \\
\text { ideal e todos os nutrientes necessários para o crescimento e desenvolvimento do lactente, } \\
\text { constituindo um dos pilares fundamentais para a promoção da saúde das crianças em todo o mundo }\end{array}$ \\
\hline 20 & $\begin{array}{l}\text { LOPES } \quad \text { et } \quad a l \\
(2018)\end{array}$ & $\begin{array}{l}\text { Analisar a prevalência do aleitamento } \\
\text { materno e a introdução de alimentos } \\
\text { complementares para crianças de zero a } 24 \\
\text { meses de idade. }\end{array}$ & $\begin{array}{l}\text { Este é um estudo transversal de base } \\
\text { populacional de crianças com menos de } 24 \\
\text { meses em Montes Claros, Minas Gerais, } \\
\text { Brasil. Os dados foram coletados em 2015, } \\
\text { por meio de entrevistas com responsáveis } \\
\text { pelo cuidado infantil na casa. O questionário } \\
\text { aplicado avaliou a situação sociodemográfica } \\
\text { da família, características maternas e infantis } \\
\text { e hábitos de consumo alimentar. A análise de } \\
\text { sobrevida foi usada para calcular a mediana } \\
\text { da prevalência e duração da amamentação e a } \\
\text { introdução da alimentação complementar. }\end{array}$ & $\begin{array}{l}\text { Constatou-se neste estudo a introdução de alimentos ultraprocessados precocemente na dieta } \\
\text { infantil, prática inadequada nos primeiros anos de vida e que reflete o padrão dietético } \\
\text { contemporâneo. }\end{array}$ \\
\hline
\end{tabular}

Fonte: Autores. 
Partindo da análise dos artigos selecionados, os estudos foram divididos em duas categorias, conforme a Tabela 1 e que serviram como orientadoras para a apresentação e discussão dos resultados.

Tabela 1: Categorias dos tipos de estudo.

\begin{tabular}{lc}
\hline Estudos abordados & Número de artigos encontrados \\
\hline$>\quad 3.1$ Alimentação infantil: relação entre o leite materno, & 10 \\
desmame precoce e desenvolvimento adequado da criança. & \\
& 10 \\
da criança antes de um ano de vida & \\
\hline
\end{tabular}

Fonte: Autores.

\subsection{Alimentação infantil: relação entre o leite materno, desmame precoce e desenvolvimento adequado da criança}

Entre os fatores que afetam a saúde infantil, encontra-se em destaque a alimentação, com a recomendação pelo Ministério da Saúde (MS), Organização Mundial de Saúde (OMS) e Fundo das Nações Unidas para a Infância (UNICEF) do aleitamento materno de forma exclusiva até a idade de seis meses da criança, recomendando-se que o aleitamento deve ser mantido associado a outros alimentos até o segundo ano de vida (Oliveira et al., 2015).

O aleitamento materno é sinônimo de sobrevivência para o recém-nascido, portanto um direito inato. Representando ainda "uma das maneiras mais eficientes de atender os aspectos nutricionais, imunológicos e psicológicos da criança em seu primeiro ano de vida" (Silva et al, 2016).

Há muitos argumentos potentes em favor da amamentação no peito. O bebê amamentado no peito materno recebe anticorpos da mãe que ajudam a protegê-lo contra infecções e alergias. Bebês amamentados no peito são menos propensos a infecções respiratórias e gastrintestinais do que os que tomam mamadeira.

O risco de obesidade ou excesso de peso parece ser maior entre bebês que recebem mamadeira. Estudos têm demonstrado que adultos que haviam sido amamentados exclusivamente no peito por pelo menos dois meses tinham um nível de colesterol mais baixo do que os que receberam qualquer outro tipo de alimentação (Oliveira, \& Fanaro, 2015).

Inúmeras pesquisas têm demonstrado a relevância do aleitamento materno exclusivo para a saúde tanto da mãe quanto do bebê. De acordo com a Organização Mundial de Saúde (OMS), o ato da amamentação tem contribuído para salvar a vida de 6 milhões de crianças a cada ano. Isto deixa claras as evidências epidemiológicas que demonstram os efeitos benéficos do aleitamento materno para a criança, para a mãe e também para a sociedade (Silva, \& Gubert, 2010).

Desde a reunião conjunta de 1979 para tratar da alimentação infantil que a OMS/UNICEF recomenda que o lactante seja amamentado exclusivamente até o $6^{\circ}$ mês para, só a partir daí, receber a complementação de outros alimentos. Outros organismos internacionais têm feito recomendações similares (Silva, \& Gubert, 2010).

Pesquisa realizada no Brasil no ano de 2013 comprovou que, em nível nacional, o aleitamento materno exclusivo do nascimento até os 3 meses de idade é registrado por um número elevado de primíparas, embora após os 3 meses este número caia, apresentando um percentual bem menor. Aponta ainda evidências das vantagens de não se introduzir a alimentação complementar antes que o bebê complete seis meses de vida e aponta dentre as desvantagens, relacionadas a administração alimentar artificial antes dos 6 meses, o retardo no crescimento e o aumento no risco de mortalidade infantil. Assim, recomenda que as crianças nascidas dentro do período normal de gestação e com peso normal devem ser exclusivamente amamentadas no seio até os seis meses de idade (Amaral, Sales, Carvalho, Cruz, Azevedo., \& et al, 2015). 
Dentre as situações que podem indicar necessidade de antecipação da introdução alimentar, encontram-se registram o baixo peso ao nascer, a prematuridade e a evolução insatisfatória do crescimento e do desenvolvimento da criança (Amaral et al, 2015).

A partir de uma Pesquisa Nacional de Saúde e Nutrição, elaborada pelo Ministério da Saúde com apoio do Fundo Internacional das Nações Unidas para a Infância - UNICEF, foi possível traçar um perfil do aleitamento materno em todo o Brasil. Na pesquisa, investigou-se a situação do aleitamento materno numa amostra de 6015 crianças com idade inferior a 47 meses. Os resultados mostraram que $97 \%$ das crianças iniciam a amamentação logo após o parto. A proporção de crianças parcialmente desmamadas foi de $43 \%$ aos três meses e $61 \%$ aos seis meses. A duração mediana do aleitamento materno foi de 134 dias para todo o Brasil. Observou-se um comportamento distinto entre população da zona rural. A população urbana teve uma duração mediana de 123 dias enquanto que a população da zona rural apresentou uma duração de 191 dias. A pesquisa apontou ainda uma duração mediana de 72 dias para o aleitamento materno exclusivo (FAPEMIG, 2002).

A Organização Mundial de Saúde (OMS) e o Fundo Internacional das Nações Unidas para a Infância (UNICEF) definiram o desmame como "Um processo pelo qual outros alimentos são introduzidos gradualmente na dieta da criança, primeiro para complementar o leite do peito e progressivamente para substituí-lo e adaptar a criança a alimentação do adulto", afirmam que até o início do século 20, as crianças não recebiam nenhuma alimentação a não ser o leite materno antes de completar um ano de vida. A tendência a introduzir outros alimentos além do leite materno antes dos seis meses de vida começou neste século e atingiu seu auge nos anos 1960, quando a mulher passou a se sentir mais emancipada e correu para o mercado de trabalho (Dias et al., 2010).

Embora muito discutida, a definição da idade para iniciar a introdução da alimentação complementar ao aleitamento materno ainda tem sido motivo de debate, principalmente, em se tratando de países em desenvolvimento.

Para Schincaglia, Oliveira, Sousa, \& Martins, 2015, leite materno é um alimento completo do ponto de vista nutricional e imunológico, suprindo as necessidades das crianças nos primeiros seis meses de vida, época ideal para o desmame, quando o bebê atingir o desenvolvimento fisiológico adequado para que possa receber alimentos sólidos, o que ocorre em função do desaparecimento do reflexo de protrusão, assim como da maturação do sistema gastrointestinal e renal, favorecendo assim a oportunidade das mães oferecerem outros alimentos ao bebê. Do ponto de vista científico, a definição do período adequado para iniciar a introdução da alimentação complementar deve levar em conta o amadurecimento fisiológico e neurológico da criança além de suas necessidades nutricionais.

A introdução precoce de alimentação complementar associa-se a elevação de risco e da frequência de disfunções gastrointestinais, o que ocorre em razão da redução dos aspectos protetores que são comuns ao leite materno (Schincaglia et al., 2015).

A nutrição insatisfatória e infecção gastrointestinal em decorrência de contaminantes existentes nos alimentos que não são adequadamente higienizados, infecções respiratórias, assim como comprometimento do crescimento e desenvolvimento adequados são variáveis relacionadas a ocorrência do desmame precoce e a realização do uso de outros alimentos pela mãe antes da idade recomendada (Schincaglia et al., 2015).

O leite de vaca tem sido o alimento mais utilizado como alternativa para substituir o leite materno, mas não é recomendado, principalmente no primeiro ano de vida, devido sua proteína ser considerada como o componente dietético mais associado a problemas alimentares registrados em bebês. Fato que não ocorre com tanta frequência em crianças com mais de dois anos de vida, visto que há tolerância progressiva à proteína do leite da vaca (Campagnolo, Louzada, Silveira, \& Vitolo, 2012). 


\subsection{Efeitos da introdução do leite de vaca na alimentação da criança antes de um ano de vida}

Estudo desenvolvido por José et al., (2016), comprovou que, por considerarem que o leite de vaca pode substituir o leite materno, muitas mães acabam adotando esta prática alimentar para seus filhos antes deles completarem seis meses de vida. Prática que pode prejudicar a saúde e o desenvolvimento adequado da criança uma vez que, além de não substituir o leite materno, ainda pode causar alergias alimentares.

O uso do leite materno antes da idade de seis meses influencia o desmame precoce, ocasionando prejuízo à saúde do bebê que, em razão da imaturidade fisiológica, pode desenvolver alergias e intolerâncias alimentares (Schincaglia et al., 2015).

Microrganismos como o campylobacter, a salmonella, o mycobacterium bovis, dentre outros, presente no leite de vaca podem acarretar o risco de enfermidades como as enterites agudas, intoxicação alimentar tuberculose e até mesmo choque anafilático em pessoas com intolerância a lactose. Doenças que, no caso de crianças menores de um ano pode levar ao óbito, enquanto o leite materno atua como protetor intestinal, produzindo um meio ácido em nível gastrointestinal que inibe o crescimento de organismos patogênicos, aumenta a sistema imunológico, estimula o desenvolvimento adequado, não provoca alergias, nem gera custos, além de contribuir para a formação da arcada dentária (Blumer, 2015).

Uma outra questão a ser mencionada é a relação do leite de vaca com a anemia ferropriva na infância. Diversos estudos têm indicado que a maior prevalência da anemia é registrada em crianças com idade entre 6 e 11 meses e que "a maior prevalência de anemia nessa faixa etária deve-se, provavelmente, a fatores de risco, como o desmame precoce com introdução de leite de vaca". Isto porque, como o leite de vaca tem aproximadamente quatro vezes mais cálcio que o leite humano, dificulta a absorção de ferro (Leal et al., 2011).

O consumo de leite de vaca pelas crianças menores de um ano pode estar associado às perdas de sangue oculto nas fezes, além de constituir um risco para o desenvolvimento da anemia. Estudos também têm evidenciado associação entre o desmame precoce e a introdução do leite de vaca na alimentação dos bebês com o desenvolvimento do Diabetes Mellitus tipo 1 (Leal et al., 2011).

Uma vez que o leite materno contém nutrientes essenciais para garantir a saúde nutricional das crianças, tais como: proteínas, imunoglobulinas, carboidratos, lipídios, calcio, fósforo, ferro, vitaminas, a OMS (2013), recomenda que os bebês, até os seis meses de vida, sejam alimentados exclusivamente com leite humano e de preferência no peito materno, pois o leite materno preenche todas as necessidades do recém-nascido em termos de vitaminas, proteínas, gordura, açúcar, água, minerais, entre outros. Alguns em menor concentração como o ferro, contudo é mais do que adequado para o crescimento do lactente. $\mathrm{O}$ mesmo não ocorre com o leite de vaca.

As diferenças entre o leite humano e o leite de vaca são significativas, tanto do ponto de vista quantitativo quanto qualitativo, determinando diferenças na nutrição e proteção do bebê, bem como no crescimento e desenvolvimento do lactente. Embora o leite de vaca e o leite humano tenham em sua composição a mesma quantidade de ferro, a absorção e a biodisponibilidade deste no leite humano é muito maior no trato intestinal do lactente. A deficiência de ferro é rara em crianças que amamentam exclusivamente nos primeiros seis meses, porém a introdução de outros alimentos neste período como o leite de vaca podem reduzir a absorção (Blumer, 2015).

Estudo transversal, desenvolvido durante a Campanha Nacional de Vacinação contra Poliomielite 2012 com o objetivo de verificar o tipo de leite consumido por crianças menores de um ano de idade e identificar variáveis associadas ao consumo de leite não materno (LNM) - fórmula infantil ou leite de vaca (LV), quando 935 acompanhantes de crianças menores de um ano responderam a um questionário estruturado sobre a alimentação da criança nas últimas 24 horas, indicaram que o consumo de leite materno e fórmula infantil foi maior entre as crianças menores de 6 meses, enquanto o de leite de vaca foi maior entre as crianças de 6 a 11 meses. A maior parte das crianças que receberam leite de vaca precocemente (antes do primeiro ano de vida) 
pertenciam às famílias de menor nível socioeconômico e inseridas em programa social específico para recebimento de leite. Os resultados do estudo sugeriram que os programas sociais de distribuição de leite estão influenciando o desmame precoce (Saldan ., \& et al. 2017).

De acordo com o estudo desenvolvido por Lopes., et al (2018), constatou-se que as práticas alimentares inadequadas e precoces podem comprometer a saúde da criança a longo e curto prazo. Diante disto, ressalta a importância da melhoria na promoção ao aleitamento materno e serviços materno-infantis visando mudar a realidade em questão.

\section{Conclusão}

Por meio da análise dos trabalhos existentes, os resultados mostraram que no Brasil, ainda que leite materno seja recomendado universalmente como fonte adequada de nutrição infantil, contribuindo significativamente para ingestão de energia e micronutrientes no primeiro ano de vida, as práticas nutricionais analisadas vão em desacordo com as recomendações dos órgãos nacionais e internacionais de saúde, há uma grande prevalência na introdução precoce de outros alimentos em substituição ao leite materno, sendo o leite de vaca o alimento mais utilizado.

Entretanto, o leite de vaca, não é o mais recomendável para a alimentação da criança antes de um ano de vida, pois além de não suprir as necessidades nutricionais da criança pode desencadear alergias e intolerâncias alimentares, anemia ferropriva, doenças crônicas como obesidade e diabetes mellitus.

A anemia ferropriva é uma das consequências mais graves e frequentes entre as crianças que fazem uso do leite de vaca antes de um ano de vida, uma vez que sua ingestão interfere na absorção de nutrientes importantes existentes no leite materno como o ferro e o zinco. Microorganismos presentes no leite de vaca, mesmo no pasteurizado, também podem acarretar enfermidades graves como enterites agudas, intoxicação alimentar e até mesmo choque anafilático em pessoas com intolerância à lactose. Sugere-se a realização de novos estudos com abordagem clínico-epidemiológica para esclarecer a temática do estudo.

\section{Referências}

Agostini, C., Decsi, T., Fewtrell, M., Goulet, O., Kolacek, S., \& et al. (2008). Documento de posicionamento médico - alimentação complementar: um comentário do Comitê de Nutrição da ESPGHAN. J Pediatr Gastroenterol Nutr, 46: 99-110.

Amaral, L. J. X., Sales, S. S., Carvalho, D. P. S. R. P., Cruz, G. K. P., Azevedo, I. C., \& et al. (2015) Benefícios do aleitamento materno no crescimento e desenvolvimento infantil: uma revisão sistemática. Revista das Ciências da Saúde do Oeste Baiano - Higia, 1(2):148-163.

Amaral, L. J. X., Sales, S. S., Carvalho, D. P. S. R. P., Cruz, G. K. P., Azevedo, I. C., \& et al. (2015). Fatores que influenciam na interrupção do aleitamento materno exclusivo em nutrizes. Rev Gaúcha Enferm, 36:127-34.

Brasil. (2014). Ministério da Saúde. Guia alimentar para a população brasileira: Promovendo a alimentação saudável. Secretaria de Atenção à Saúde. Coordenação-Geral da Política. 236p. - Série A Normas e Manuais Técnicos. Brasília.

Campagnolo, P. D. B., Louzada, M. L. C., Silveira, E. L., \& Vitolo, M. R (2012). Práticas alimentares no primeiro ano de vida e fatores associados em amostra representativa da cidade de Porto Alegre, Rio Grande do Sul. Rev Nutr, 25(4):431-439.

Cunha, A. J., Leite, A. J., \& Almeida, I. S. (2015). O papel do pediatra nos primeiros mil dias da criança: a busca de uma nutrição e desenvolvimento saudáveis. J Pediatr, 91:44-51.

Dias, M. C. A. P., Freire, L. M. S., \& Franceschini, S. C. C. (2010). Recomendações para alimentação complementar de crianças menores de dois anos. Rev. Nutr, 23(3)

FAPEMIG. (2002). Intoxicação alimentar. Revista Minas Faz Ciência [série eletrônica], 11.

José, D. K. B., Vitiato, J.A., Hass, K., França, T. C. S., \& Cavagnari, M. A. V. (2016). Relação entre desmame precoce e alergias alimentares. Visão Acadêmica, Curitiba, 17(3).

Leal, L. P., Batista Filho. M., Lira, P. I. C., Figueiroa, J. N., \& Osório, M. M. (2011) As doenças relacionadas ao consumo de leite de vaca.

Leal, L. P., Batista Filho. M., Lira, P. I. C., Figueiroa, J. N., \& Osório, M. M. (2011). Prevalência da anemia e fatores associados em crianças de seis a 59 meses de Pernambuco. Rev. Saúde Pública, 45(3). 
Research, Society and Development, v. 11, n. 3, e41311325554, 2022

(CC BY 4.0) | ISSN 2525-3409 | DOI: http://dx.doi.org/10.33448/rsd-v11i3.25554

Lopes,W. C., et al. (2018). Alimentação de crianças nos primeiros dois anos de vida. Rev. paul. pediatr, 36(2).

Mendes, K. D. S., Silveira, R. C. C. P., \& Galvão, C. M. (2012). Revisão integrativa: método de pesquisa para a incorporação de evidências na saúde e na enfermagem. Texto Contexto Enferm, Florianópolis, 17(4): 758-64.

Mendonça, R. B., Cocco, R. R., Sami, R. O. S., \& Solé,D. (2011). Teste de provocação oral aberto na confirmação de alergia ao leite de vaca mediada por IgE: qual seu valor na prática clínica? Rev Paul Pediatr, 29(3):415-22.

Oliveira, M. F., \& Fanaro, B. F. (2015). Aleitamento materno na prevenção de sobrepeso, obesidade infantil e alergias. Revista Brasileira de Nutrição Clínica. São Paulo, 30(3): 216-26.

Organização Mundial da Saúde. (2013). Ações nutricionais essenciais: melhorar a saúde e nutrição materna, neonatal, infantil e infantil. Genebra: OMS, PubMed. Organização Mundial de Saúde. (2013). Tendências e níveis de mortalidade infantil - Relatório 2013. Genebra: OMS - Série de Informes Técnicos.

Passanha, A., Cervato, M. A. M., \& Silva, M. E. M. P. (2010). Elementos protetores do leite materno na prevenção de doenças gastrintestinais e respiratórias. Rev. Bras. Cresc. e Desenv. Hum, 20(2): 351-360.

Pereira, P. F., Alfenas, R. C., Araújo, R. M. A. (2014). O aleitamento materno influencia o risco de desenvolvimento de diabe tes mellitus na criança? Uma análise das evidências atuais. J. Pediatr, 90(1).

Saldan, P. C., \& et al. (2017). Consumo de leite em menores de um ano de idade e variáveis associadas ao consumo de leite não materno. Rev. paul. pediatr, 35(4): 407-414

Schincaglia, R. M., Oliveira, A. C., Sousa, L. M., \& Martins, K. A. (2015). Práticas alimentares e fatores associados à introdução precoce da alimentação complementar entre crianças menores de seis meses na região noroeste de Goiânia. Epidemiol. Serv. Saúde, Brasília, 24(3):465-474.

Schincaglia, R. M., Oliveira, A. C., Sousa, L. M., \& Martins, K. A. (2015).. Prevalência do início do desmame precoce em duas populações assistidas por serviços de puericultura de Florianópolis, SC, Brasil. Pesq Bras Odontoped Clin Integr, 11(2):239-44.

Silva, R. Q., \& Gubert, M. B. (2010). Qualidade das informações sobre aleitamento materno e alimentação complementar em sites brasileiros de profissionais de saúde disponíveis na internet. Rev. Bras. Saúde Matern. Infant. Recife, 10(3): 331-340.

Sociedade Brasileira de Pediatria. (2012). Manual de orientação para a alimentação do lactente, do pré-escolar, do escolar, do adolescente e na escola. 3. ed. Rio de Janeiro: SBP, Departamento de Nutrologia.

Victora, C. G., \& et al. (2016). Amamentação no século 21: epidemiologia, mecanismos e efeitos vitalícios. Lanceta, 387: 475-490.

Walker, S. P., Wachs, D. T., Gardner, J. M., Lozoff, B., Wasserman, G. A., \& Pollitt, E. (2007). Desenvolvimento infantil: fatores de risco para desfechos adversos em países em desenvolvimento. Lanceta, 369: 145-157. 